empirical and exclusive application of a single instrument, into an art susceptible of analysis and development; capable of furnishing the beneficent hand of the surgeon with varied means for the relief of aflicted humanity, and which may, I hope, now be ranked amongst the most positive subjects of study.

\section{FXTERNAL APPLICATION OF QUININE IN AGUE.}

\section{To the Editor of THE LANCET.}

Sir, - Through the medium of your widely-circulating periodical, I wish to direct the attention of the profession to a fact of some importance in thie treatment of intermittent fever. It is this:-cases of ague sometimes occur, in which it is unadvisable or impossible to administer the sulphate of quinia internally. Under such circumstances, I have seen the following treatment attended with the best effects :$A$ blister is applied over some part of the epigastric region; when it bas risen, the cuticle is removed, and the surface ligbtly sprinkled with the sulphate of quinia, and dressed with some simple ointment. Absorption of the quinia takes place, and thus the cure is accomplished.

I have seen this plan adopted in several instances with general, but not invariable, success; but neither is the quinia treat. ment in the other forms, when admissible, invariably successful. I think it but right to state, that in most, but not in all, instances, this application is attended with considerable pain for some hours, and in one case there was a superficial ulcer produced by sloughing of the cutis. I would suggest, but have not tried, poulticing the blistered surface for the first twelve or twenty-four hours after removal of the cuticle, and then applying the sulphate of quinia in the form of ointment, combined with opium or bella. donna, \&c., to obviate pain.

This treatment $I$ have never seen in print, and first heard of it in the hospital of this town, where the experiments were made at a period when ague was prevalent in the neighbourhood. If it is of any value, which can only be decided by longer experience, I must acknowledge that to my scientific friend Dr. Purcell, who is at the head of that institution, is due the merit of suggesting it. I am, Sir, yours, \&c.,

Thomas Christin.

Carrick-on-Suir, Ireland, Feb, 13, 1833 .

No. 495.

\section{STATISTICAL REMARKS}

ON THE EFFECTS OF

\section{H OLERA I N FRA N C E}

DURING THE EPIDEMIC OF 1832.

By A. Moreau de Jonnes.*

1st. THE duration of the irruption of cholera in France extended from March 15 th to December 31 st, 1832 , a period of 291 days, or nine months and a half. At the end of that period, the disease still existed in twelpe departments, notwith. standing the diminished temperature.

2nd. The departments in which the cholera continued from the beginning to the end of that long irruption, are those of the Pas de Calais and Seine.

3rd. The disease attacked 50 departments successively, viz.

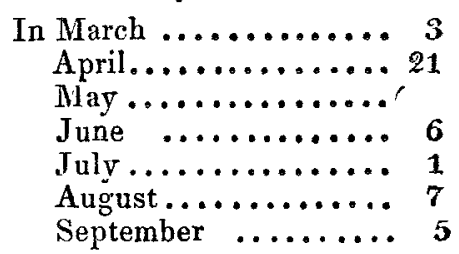

4th. The entire surface of these 50 de. partments is 16,267 square leagues, which leaves 10,783 for the 36 departments not attacked by cholera in 1832.

5 th. But in marking the degree of the infection, the area overrun by the cholera must be limited. Nineteen departments, containing 5840 square leagues, were entirely overrun; twenty-one departments, containing 7228 square leagues, bad not a third of their population infected. Forty departments, then, containing 13,068 square leagues, suffered severely from the disease. Ten other departments, containing 3199 square leagues, suffered but slightly.

6 th. Leaving out these departments, we may conclude, generally, that the cholera in nine months and a half of 1832 , has spread over about half the surface of France.

7 th. The disease spread by contiguity, as in the other parts of Europe, following lines, of which Paris was the centre, ramifying with the communications through the country.

8th. In each department the time of the irruption was sooner or later after that of Paris, in proportion to the distance from, and the frequency and rapidity of the communications with, the capital. Thus the departments of the east became infected in the following dates, viz.

* Communicated by Sir David Barry. 
Seine ............ NTarch 24 .

Seine and Marne .... April 2.

Marne ............ 11 .

Meuse $\quad . . \ldots \ldots \ldots . . .16-16$.

Moselle .......... -27 .

Meurthe $\ldots . . . .$. May 4.

Vosgez ........... 13 .

Haute Saone ........ June 16 .

In the western departments, the disease broke out as follows:-

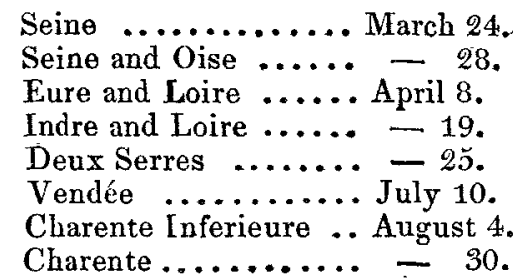

The cholera, which got into the departments of the south by the navigation of the Rhone, did not appear there before the end of August, or in the course of September; one hundred days after Paris had been attacked.

9th. The mortality produced by cholera, divides the infected departments into three series, viz.-The first comprehends those where the deaths were above 1000 . The second, those where the deaths were from 100 to $1000^{\circ}$. The third, those in which the official returns give under 100 deaths.

10 th and 11th. Recapitulation of these series. Omitted.

12th. The degree of rapidity with which the disease spread in different directions is as follows :-Cholera appeared in Calais on the 15th of March 189\%, and broke out at Arlis on the 17th of September following, Laving thus, in 186 days, traversed 200 leagues, forming the great diameter of France from north to south.

The disease was recognised in Paris, the eentre of the kingdom, on the 24th of March. On the 27th of April following, it had spread by contiguity to the department of the Moselle, and on the 11th of May to that of Finisterre, taking thirty-five days to reach the eastern, and fifty days to arrive at the western, frontier of France, having traversed on the one side seventy leagues, and on the other 120 .

Thus the cholera bas traversed the territory of the kingdom from north to south at the rate of one league in twenty-four loours, whilst, from east to west, it required but eighty-five days to travel a distance of $\mathbf{1 9 0}$ leagues, which gives a rapidity of spread greater by one-half.

13th. The progress and the decline of the disease, made out at the end of each month, are as follows :-
Persons attacked. Deaths.

April .......38,005 ....16,607

May .......31,388...11,599

June $\ldots \ldots \ldots 47,916 \ldots \ldots 19,440$

July .......48,419...18,632

August $\ldots \ldots .33,041 \ldots \ldots 13,321$

September ....19,859....9,049

October ..... 8,410... 9,689

November .... 4,225 ... 1,727

December..... 1,270... 602

$$
\overline{229,533} \overline{94,666}
$$

Thus the cholera attained the maximum of its effects during the fourth month of its invasion. Its power of propagation afterwards diminished from month to month with the diminution of the temperature.

14 th. Still the violence of the disease was not diminished by any atmospheric influence ; for during the tenth month of its irruption, as at the time of its first appearance, one-half of those attacked died. The official documents give a total of three deaths in seven attacks. But it is notorious that in many cases, deaths from cholera were set down to ordinary diseases. For example, in Paris, the deaths caused by cholera, or its immediate consequences, during 160 days, were diminished by $\mathbf{1 7 0 8}$, or nearly onetenth of the whole.

15th. Admitting these corrections and some others, the minimum of the mortality from cholera in France may be estimated at 110,000 deaths, or more than a 300rdth of the population.

The mortality from cholera in the different countries of Europe that have suffered from the disease, has been in the following proportions, viz.

In Russia (two irruptions) $1-20$ th

Austria .......... 1-30th

Poland ............ 1-32nd

Prussia (twoirruptions) 1-100th

Belgium .........1.120th

Great Britain and Ire-

land ............1.131st

Holland.......... 1-144th

Germany (in the infected parts $). . \ldots \ldots$ 1-700th

16th. The average of the ordinary mortality in France, during 1829 and 1830 , having been $8 \% 1,934$, the effects of the cholera will have augmented that of 1832 by one-eighth, and during this last year, the population will have had scarcely any increase by the excess of births over deatbs ; that excess in Paris in 1830 having been only 1121. It will take twenty years to. repair the loss of 20,300 indiriduals carried off by cholera in the space of 283 days. 